\title{
An Apparatus for the Growth of Metal Single Crystals
}

Cite as: Review of Scientific Instruments 4, 656 (1933); https://doi.org/10.1063/1.1749053 Submitted: 28 August 1933 . Published Online: 27 December 2004

Maurice F. Hasler
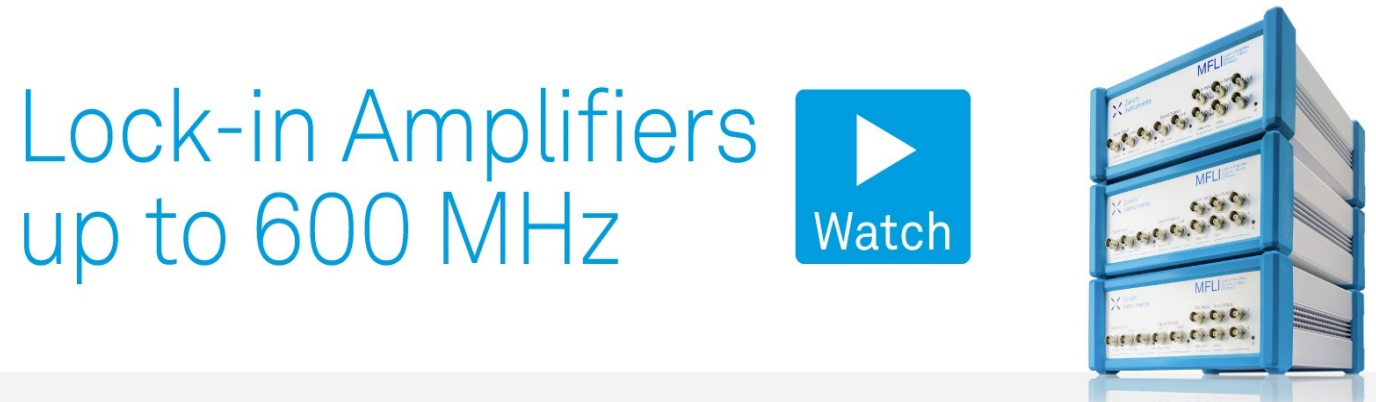


\title{
An Apparatus for the Growth of Metal Single Crystals
}

\author{
Matrice F. Hasler, Norman Bridge Laboratory of Physics, California Institute of Technology
}

(Received August 28, 1933)

A simple crystal growing apparatus is described which allows the production of very uniform single crystalline rods of low melting point metals. The crystals may be produced in any desired gas atmosphere or in a vacuum. Any orientation of crystal to rod may be obtained by a simple seeding procedure.

\section{INTRODUCTION}

$M^{A}$ ANY methods for growing metal single crystalline rods have been described to date, ${ }^{1}$ though the resultant crystals have not always possessed all of the following properties to a high degree; uniformity of shape, size, composition, and orientation throughout the lengths of the crystals; freedom from strains; and any desired crystallographic orientation. These methods do, however, bring out the factors that influence crystal growth, namely, velocity of growth, the temperature gradient in the crystallizing region and the stresses applied to the crystal. The optimum values for these various factors will be discussed with respect to the apparatus described herein.

\section{Casting and Growing Mouldo}

Producing crystals of precise shape, size, and composition requires two processes; the casting of a polycrystalline bar possessing these characteristics, and the growing of the final single crystal from the polycrystalline bar. For the casting moulds Acheson graphite is used as

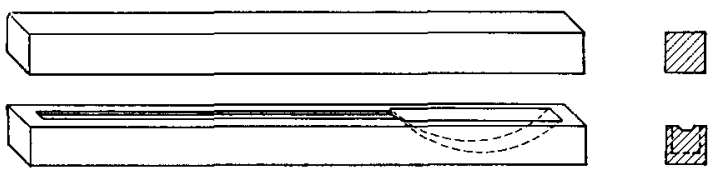

FIG. 1. Combination casting mould and crucible.

this material is quite pure, does not react with metals at low temperatures, is easily machined and may be outgassed if crystals are to be produced in a high vacuum. Fig. 1 shows the two halves of the mould which are clamped together after the metal is placed within the cavity which acts as a melting crucible. The moulding space may have any desired cross section, that illustrated being a trapezoid and particularly suitable for metals which expand upon solidification. The mould is placed within a Pyrex tube which may either be evacuated or filled with a non-oxidizing gas. The whole is then heated to about $50^{\circ}$ above the melting point of the metal with either an electric furnace or an induction furnace, the latter being effective in stirring the melt when alloys are being prepared. Temperatures are measured by a thermocouple fitted into a hole in the lower half of the mould. The bar is cast by merely tipping the tube with its mould which is then allowed to cool. Bright homogeneous castings accurately filling the slot are thus obtained.

For the final growth of the crystal another mould must be prepared. This is probably one of the most important parts of the whole apparatus to be described as upon it depends to a large measure, the heat gradient in the crystallizing region of the metal bar and the stresses applied to this region, two of the three important factors in crystal growth. Acheson graphite is also used for this trough, Fig. 2a showing its geometry. The space which holds the crystal must have a cross section that is identical with that of the mould used in casting the polycrystalline bar. The amount of material surrounding this space determines to a large extent the heat gradient within the crystal. For the growth of bismuth crystals, for instance, a very steep heat gradient is required and thus a mould cross section as indicated in Fig. $2 \mathrm{~b}$ is suitable. Such a shape insures sufficient rigidity to the mould and yet makes for small conduction along the mould, while assisting radial 


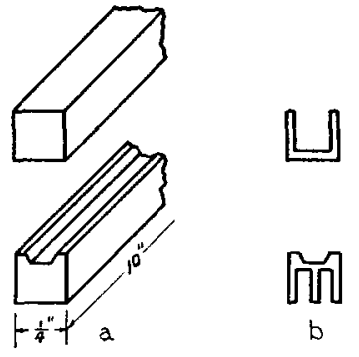

Fig. 2. (a) Growing trough. (b) A modified cross section for a growing trough.

conduction, especially to the glass floor on which the trough rests during the crystal growing process. Another consideration is the weight of the cover. For materials which expand upon solidification this must be kept sufficiently small to prevent stressing the forming crystal. For materials which contract this is not important providing that the polycrystalline rod does not more than fill the mould. On the other hand the cover must not be so light in weight that surface tension forces of the metal bar may lift it and thus destroy the perfection of crystal cross section. It should be emphasized that growing successful crystals depends upon having these factors, the heat conduction along the trough and the weight of the cover, just right. In fact, time spent in experimenting with different troughs is more than repaid in time saved when a large number of successful crystals must be grown.

\section{Details of the Crystal Growing Apparatus}

The crystal growing apparatus proper is shown schematically in Fig. 3. $A$ is a cylindrical electric furnace 10 inches in length and 4 inches in diameter with a $1 \frac{1}{2}$ inch opening through the center. The heating element is a non-inductive No. 20 chromel wire winding. The dimensions of the furnace chosen depend entirely upon the size and characteristics of the crystals to be grown. The furnace mentioned above allows pieces of metal up to 10 inches in length to have the whole uncrystallized part molten throughout the entire growing process. This is important as it allows the cover of the crystal growing mould to settle down against the lower piece thus insuring a perfect cross section for the final crystal. $B$ is a gear box which has a ratio of 125,000 to 1 . It is driven by a $1 / 30$ horse power d.c. shunt wound motor the speed of which may be controlled by adjusting the voltage in the armature circuit. Motor speeds between 100 and 1725 r.p.m. are thus easily obtained. $C$ is a $4.7 \mathrm{~cm}$ driving wheel of the gear box to which is fastened a length of chain which passes over the pulley $P_{1}$ and connects to the furnace $A$. Another chain fastened at the right side of the furnace passes over the pulley $P_{2}$ and is weighted at $W$. Three wheels at $R_{1}, R_{2}$ and $R_{3}$ moving on tracks $T_{1}$ and $T_{2}$ allow motion of the furnace. $R_{1}$ and $R_{2}$ are grooved and move on the ridge of $T_{1}$ which possesses a triangular cross section. $R_{3}$ is flat and moves on the flat track $T_{2}$. This manner of support eliminates wheel levelling and aligning difficulties completely. Thus as $C$ pays out chain, the furnace moves from left to right at a very uniform rate which may be varied between $0.0002 \mathrm{~cm} / \mathrm{sec}$. and $0.0034 \mathrm{~cm} / \mathrm{sec} . D$ is a glass

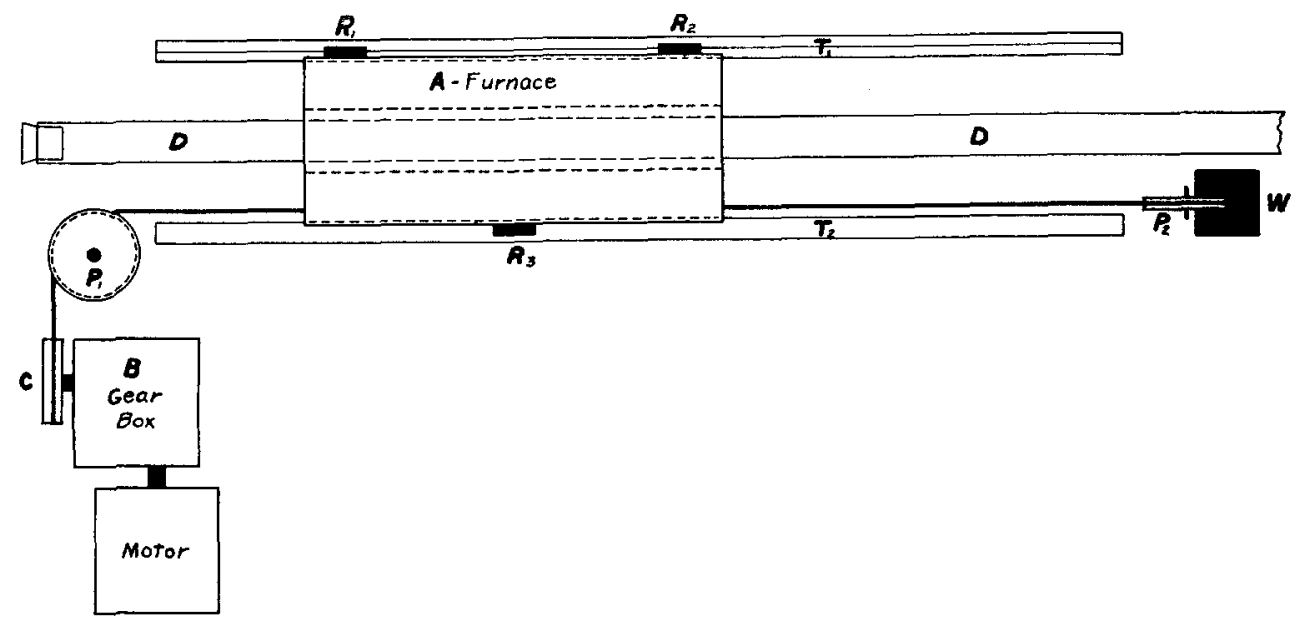

FIG. 3. Schematic diagram of the crystal growing apparatus. 
tube approximately $1^{\prime \prime}$ in diameter with $1 / 32^{\prime \prime}$ thick walls. This is rather thin walled tubing and is so chosen to allow a steep heat gradient in the graphite growing trough which is placed within it. This tube passes through the center of the furnace and is supported from a beam which is mounted parallel to it above all the apparatus. It may be seen at the top of Fig. 4

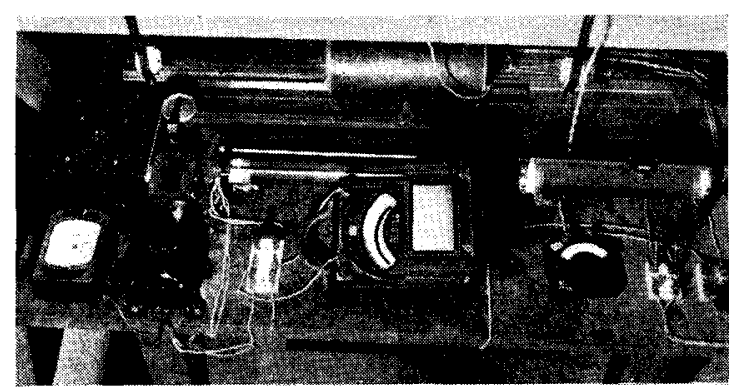

FIG. 4. Photograph of crystal growing apparatus.

which is a photograph of the crystal growing apparatus. This beam is mounted upon supports which are fastened to the floor and are independent of the table upon which the rest of the apparatus is mounted. Thus any vibrations caused by the motor cannot be transmitted to the growing crystal in its graphite trough within the glass tube. Any gas atmosphere may be maintained about the crystal by filling the tube. A vacuum obtained with a fore-vacuum pump was usually used in this apparatus. For a really high vacuum a ground glass stopper could be placed at the left side of the tube where the crystal is admitted and a diffusion pump connected at the right side.

\section{Seeming of Crystals}

Without seeding, single crystals of indeterminate orientation may be grown in this apparatus. For most work, however, crystals with a predetermined orientation are necessary. These are obtained by inoculating the beginning of the polycrystalline rod with a so-called "seed," which is a piece of crystal one or two centimeters long possessing the desired crystallographic orientation. It is essential that this piece be unstrained as slipping or twinning at the seeding point may produce final crystals with different orientations than those desired. The polycrystalline bar and the seed are put end to end in a trough possessing a slot similar to that of the other moulds. This trough should be made of some very poor heat conducting material such as diatomaceous earth brick. A small hydrogen flame is then used to heat the contact between the two metal pieces. Since it reduces any oxide on the metal the two pieces run together on melting and form a good clean homogeneous joint. If the trough holding the pieces is a sufficiently poor conductor of heat this junction may be produced with the melting of only a few millimeters of the seed crystal. It should be emphasized that this joint must be well made so that no centers of crystallization apart from the main body of the crystal exist. Surface tension effects will always make this joint thicker than the polycrystalline bar. This however causes no difficulties if the cover closing the growing trough is rested from this point on over the metal bar. When the crystal is grown the joint together with the seed is removed the rest of the crystal then possessing the uniform cross section desired.

\section{Procedure for Growing Crystals}

The actual procedure for growing a crystal is almost self-evident. After a polycrystalline bar has been poured it is seeded with a piece of crystal, possessing the desired orientation, by the method described above. The bar with its attendant seed is then placed within the graphite growing mould and the cover placed over the polycrystalline part. It is essential that this cover fits well even before the bar is molten as otherwise irregularities in cross section may result. The trough is placed within the glass tube with the seed toward the opening. The system is then sealed and either a gas introduced or a vacuum produced as is desired. The furnace is moved to a position where all the polycrystalline bar is within it, only the seed and trough extending beyond the left-hand end. The furnace is then heated to a temperature such that the whole bar and a portion of the seed is molten. It is essential that the melting should occur back to a point within the seed beyond where it was previously molten so that real single-crystal inoculation is secured. The motor is then started and the crystal grows at the rate which the furnace moves down the tube. 


\section{A Specific Example}

With the apparatus described herein bismuth crystals have chiefly been grown. The fact that bismuth expands upon solidification has made it quite difficult, until the present time, to grow unstrained crystals possessing very definite cross sections. With this apparatus, however, this may be done successfully for crystals of any crystallographic orientation. The dimensions of the crystals usually grown are $1.25 \times 3 \times 120 \mathrm{~mm}$ with the cross section determined by the slot shown in Fig. 2b. Surface tension tends to round the corners somewhat, but aside from this the mould is accurately filled. Fig. 5 is a photograph of a casting and a finished crystal that has been etched to show its single crystalline form.

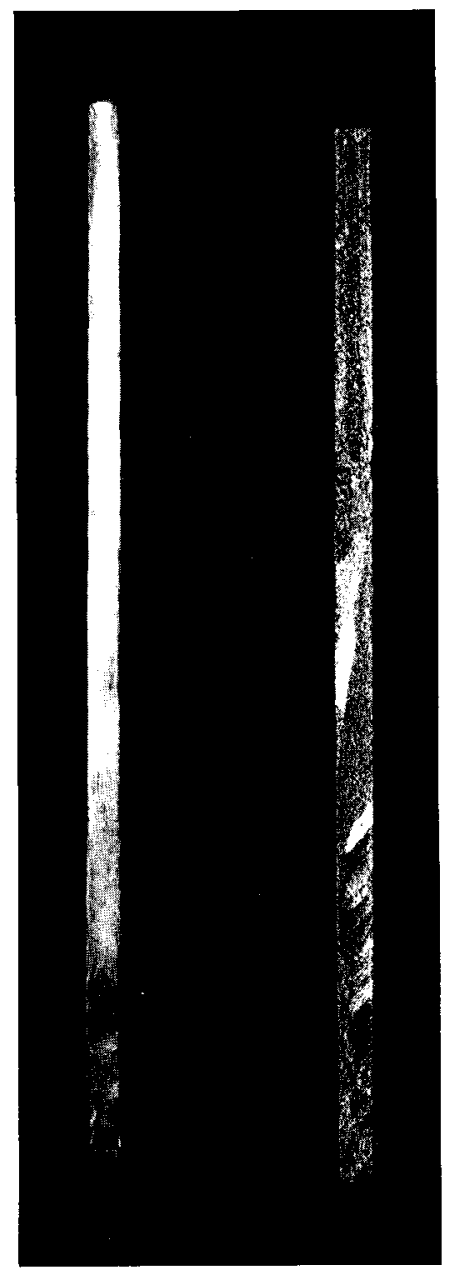

FIG. 5. Photograph of a polycrystalline casting (at right) and a single crystal with seed (at left).
Values for the three important factors influencing crystal growth, mentioned in the introduction, may be of interest in connection with these crystals. Any growing velocity less than about $0.0085 \mathrm{~cm} / \mathrm{sec}$. is found suitable for bismuth crystals. A bar of the dimensions mentioned above in the cut-away growing trough of Fig. 2b produces a temperature distribution along the crystal when growing which is shown in Fig. 6. From this it is seen that a temperature

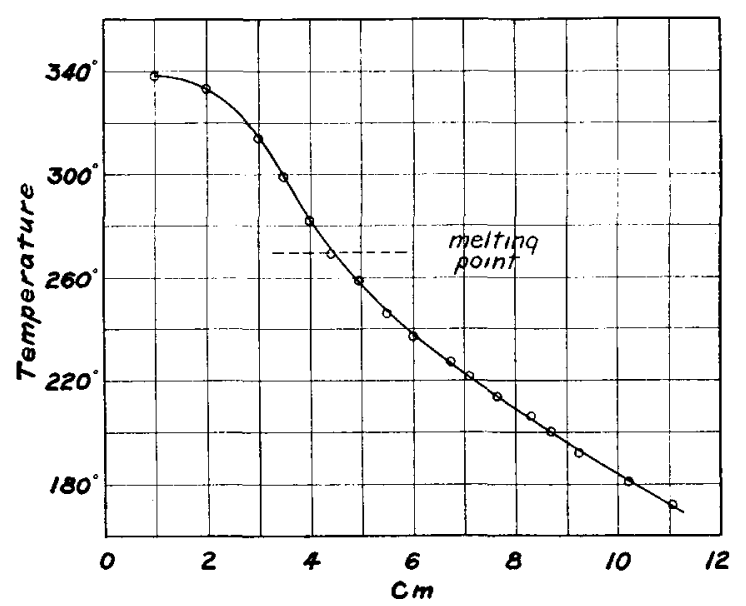

FIG. 6. Temperature distribution along a bismuth crystal which solidifies at $270^{\circ} \mathrm{C}$.

gradient of approximately $20^{\circ} \mathrm{C}$ per $\mathrm{cm}$ exists at the point of crystallization. From experiences with larger troughs which give smaller gradients it is apparent that for successfully growing bismuth crystals a large heat gradient along the crystal is required. As previously pointed out the stresses applied to the crystal depend largely upon the weight of the graphite cover. The cover used for producing these bismuth crystals weighed $12 \mathrm{~g}$ being 10 inches long.

Another factor of importance, at least in the case of bismuth, is the temperature of the melt within the furnace. If as a mass of evidence seems to indicate, ${ }^{2}$ a crystalline phase exists within the molten metal for temperatures many degrees above the melting point then to destroy any adverse orienting effects left over from the polycrystalline grains a high temperature treatment of the liquid is necessary if a single crystal is to be produced. For bismuth this region is estimated to extend $10^{\circ}$ above the melting point 
so that the $70^{\circ}$ used as shown in Fig. 6 should be ample as a "disorienting temperature."

With the apparatus and conditions described the percentage of successful crystals of those attempted has been very high. The probability of accidental disturbances occurring which might spoil the crystal is directly proportional to the time of growth so that other things being equal the more rapidly grown crystals have a better chance of being successful. For crystals grown at the rate of $0.0034 \mathrm{~cm} / \mathrm{sec}$, practically 100 percent of successes is attained. For crystals grown very slowly, $0.0003 \mathrm{~cm} / \mathrm{sec}$. Those having their trigonal axis normal to the rod have a higher success percentage than those having their trigonal axis parallel to the rod, the former having 100 percent, while the latter 70 percent. Even this latter figure is exceptionally good when it is realized that this orientation of crystal to rod is the most unstable for any growing conditions due to the large anisotropy of the physical properties of bismuth. This fact has been discussed at length in a previous paper.*

\section{General Applicability of the Method}

In order to test the general applicability of this method for the growth of single crystals of low melting point metals several others besides bismuth were tried. These, because of their possible use in this laboratory, were tin and cadmium.

For tin since the surface tension of the molten metal is larger than for bismuth more metal must be used for the first casting than is necessary to just fill the trough, to provide a "pressure head" and thus cause complete filling of the mould. For the final growth the same trough was used as for bismuth and the crystals grown in a vacuum at the rate of $0.004 \mathrm{~cm} / \mathrm{sec}$. Perfect single crystals resulted possessing excellent uniformity of shape, size, and crystallographic orientation throughout their lengths.

* A. Goetz and M. F. Hasler, Proc. Nat. Acad. 15, 646 (1929).
Cadmium due to its large surface tension and vapor pressure provided some difficulties which were, however, rapidly overcome. The casting if preformed with sufficient metal in the reservoir and with shaking of the trough was successful, the form being effectively filled. This, because it is a fairly rapid process, could be conducted in a vacuum despite the vaporization of the metal. For the final growth, however, an atmosphere of hydrogen was used to reduce the rate of evaporation. To prevent the surface tension from lifting the light cover of the growing trough it was weighted with a piece of glass tubing. With these slight modifications single crystals of cadmium were grown successfully at the rate $0.004 \mathrm{~cm} / \mathrm{sec}$. though evaporation of the material tended to make the cross section slightly indeterminate. Increasing the gas pressure in the glass tube would undoubtedly overcome this difficulty.

Up to the present no other metals have been used from which to grow single crystals though from the ease with which these crystals are grown for bismuth, tin and cadmium there is every reason to expect that equally good results may be obtained for other metals if the critical factors are well adjusted.

It is a pleasure to express my appreciation to Dr. A. Goetz for fostering this research and to Mr. Kendall for growing many crystals.

\section{BIBLIOGRAPHY}

(1) J. Czochralski, Zeits. f. physik. Chemie 22, 219 (191718).

G. Tammann, Lehrbuch der Metallographie, 1921.

E. V. Gomperz, Zeits. f. Physik 8, 184 (1922).

Obriemow and Schubikow, Zeits. f, Physik 25, 31 (1924).

P. W. Bridgman, Proc. Am. Acad. Arts and Sc. 60 , 307 (1925).

P. Kapitza, Proc. Roy. Soc. A119, 358 (1928).

A. Goetz, Phys. Rev. 35, 193 (1930).

Schubnikow, K. Acad. Amst. Proc. 33, 327 (1930).

Palibin and Froiman, Zeits. f. Krist. 85, 322 (1933).

(2) R. W. Boydston, Phys. Rev. 30, 911 (1927).

A. Goetz, Phys. Rev. 35, 193 (1930).

A. Soroos, Phys. Rev. 41, 516 (1932).

Webster, Proc. Roy. Soc. A140, 653 (1933). 